\title{
FRICTIONAL SLIP BETWEEN A LAYER AND A SUBSTRATE CAUSED BY A NORMAL LOAD
}

\author{
MARIA COMNINOU and D. SCHMUESER \\ Department of Applied Mechanics, University of Michigan, Ann Arbor, MI 48109, U.S.A. \\ and \\ J. DUNDURS \\ Department of Civil Engineering, Northwestern University, Evanston, IL 60201, U.S.A.
}

\begin{abstract}
An elastic layer that rests on a substrate and is subjected to a concentrated normal force is used to investigate some aspects of frictional slip between two bodies. The formulation using known results for glide dislocations leads to a singular integral equation of a Cauchy type that must be solved numerically. Results for various quantities of interest are given graphically.
\end{abstract}

\section{INTRODUCTION}

THE ELASTIC layer resting on a substrate provides one of the simpler and more lucid examples for a receding contact between two bodies[1]. As such it has received considerable attention[2-12], but only under the assumption that the contact is frictionless. None the less, the layer can also be used to give tractable examples for the effect of friction in contact problems. Since there are very few elasticity solutions and even fewer general results for frictional contact, it is instructive to investigate the various aspects of frictional slip between the layer and the substrate.

We consider in this article a layer that is pressed uniformly against a substrate. The initially uniform pressure between the two bodies may be due to gravity or loading at the surface of the layer. If the surface of the layer is also subjected to some other loads, the distribution of the normal tractions is modified, and the contact interface has to transmit shearing tractions. When the level of these loads is gradually raised, slip, separation or both eventually take place. In the present article we treat the loading by a concentrated force applied to the surface of the layer. We consider both a compressive and a tensile force, restricting the magnitude of the latter so that no separation occurs. For mathematical simplicity we assume that the layer and the substrate consist of identical materials.

\section{FORMULATION}

Consider a layer of thickness a pressed against the semi-infinite substrate with the same shear modulus $\mu$ and Poisson's ratio $\nu$. The initial distribution of pressure between the two bodies is uniform and is denoted by $p_{0}$. Suppose that a normal concentrated force $P$ exerting tension is applied to the surface of the layer at $x=0$, as indicated in Fig. 1, that $P=P(t)$ is a monotonically increasing function of time $t$, and that $P(0)=0$. The tractions transmitted by the interface in the bilateral problem, which places no restriction on the nature of these tractions, are obtained from the Flamant solution[13] as

$$
\begin{aligned}
& \sigma_{x y}(x, 0)=-\frac{2 P}{\pi a} \frac{a^{3} x}{\left(a^{2}+x^{2}\right)^{2}} \\
& \sigma_{y y}(x, 0)=-p_{0}+\frac{2 P}{\pi a} \frac{a^{4}}{\left(a^{2}+x^{2}\right)^{2}}
\end{aligned}
$$

These tractions are also acceptable in the unilateral problem as long as the appropriate inequalities are not violated, and neither slip nor separation occur. The conditions for stick without separation are

$$
\sigma_{y y}(x, 0)<0, \quad\left|\sigma_{x y}(x, 0)\right|<f\left|\sigma_{y y}(x, 0)\right|
$$




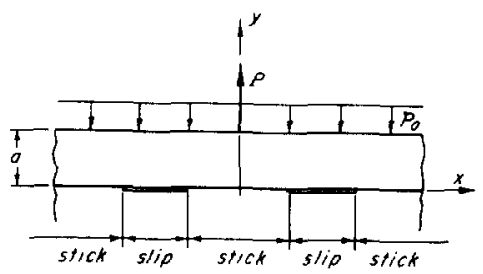

Fig. 1. Geometry of the layer and substrate, and the expected slip zones.

where $f$ is the coefficient of friction (no distinction is made here between static and kinetic friction). The inequalities show that there is no separation for

$$
P / p_{0} a<\pi / 2
$$

and that no slip takes place provided

$$
\frac{P}{p_{0} a}<\frac{8 \pi f\left[3+2 f^{2}-f\left(3+4 f^{2}\right)^{1 / 2}\right]^{2}}{27\left[f+\left(3+4 f^{2}\right)^{1 / 2}\right]}
$$

If (6) is eventually violated upon a gradual increase of the applied concentrated force $P$, slip starts at

$$
x=\frac{1}{3} a\left[\left(3+4 f^{2}\right)^{1 / 2}-2 f\right]
$$

It can be shown from (1-4) that slip begins before separation for any value of the friction coefficient $f$.

The case of a compressive concentrated force $P$ can be analyzed similarly. The condition for stick is then

$$
\frac{P}{p_{0} a}<\frac{8 \pi f\left[f\left(3+4 f^{2}\right)^{1 / 2}+3+2 f^{2}\right]^{2}}{27\left[\left(3+4 f^{2}\right)^{1 / 2}-f\right]}
$$

and slip starts at

$$
x=\frac{1}{3} a\left[\left(3+4 f^{2}\right)^{1 / 2}+2 f\right]
$$

where $P$ is reckoned positive for a compressive force. It is clear that there is no separation in this case.

In the sequel, we examine the problem of frictional slip without separation. The equations are written only for the tensile load, but numerical results will be presented for both tensile and compressive applied forces $P$.

The solution of the unilateral problem is constructed by correcting the bilateral elastic fields derived from the Flamant solution. This is done by representing the slip zones as continuous distributions of edge dislocations with Burgers vectors that lie in the interface (glide arrays). Consider for this purpose a discrete edge dislocation with the Burgers vector $b_{x}$ (slip parallel to the surface) situated at $x=\xi, y=0$ in an elastic half plane, with the coordinate axes placed exactly as in Fig. 1, so that $y=a$ is the free surface and the dislocation is at a depth $a$ from the free surface. Of interest in the formulation are only the tractions acting on the slip plane of the dislocation, or $y=0$. The needed expressions are readily extracted from the results given in Refs.[14-16]. Thus the required traction components are

$$
\begin{aligned}
& \tau_{x y}(x, 0)=\frac{2 \mu b_{x}}{\pi(\kappa+1)}\left\{\frac{1}{x-\xi}-\frac{x-\xi}{4 a^{2}+(x-\xi)^{2}}+\frac{12 a^{2}(x-\xi)}{\left[4 a^{2}+(x-\xi)^{2}\right]^{2}}-\frac{64 a^{4}(x-\xi)}{\left[4 a^{2}+(x-\xi)^{2}\right]^{3}}\right\} \\
& \tau_{y y}(x, 0)=\frac{16 \mu b_{x}}{\pi(\kappa+1)}\left\{-\frac{3 a^{3}}{\left[4 a^{2}+(x-\xi)^{2}\right]^{2}}+\frac{16 a^{5}}{\left[4 a^{2}+(x-\xi)^{2}\right]^{3}}\right\}
\end{aligned}
$$


where $\kappa=3-4 \nu$ for plane strain. The tractions induced by an array of continuously distributed edge dislocations are obtained by replacing $b_{x}$ with $B_{x}(\xi) \mathrm{d} \xi$, where $B_{x}(\xi)$ is the density of the distribution, and integrating with respect to $\xi$. The distributed dislocations lead to a tangential shift in the slip plane

$$
h(x)=v_{x}\left(x, 0^{+}\right)-v_{x}\left(x, 0^{-}\right)
$$

where $v_{\mathbf{x}}$ denotes the $x$-component of displacement corresponding to the dislocation array. The tangential shift is related to the dislocation density by

$$
B_{x}(x)=-\frac{\mathrm{d} h(x)}{\mathrm{d} x}
$$

The formulation using dislocations automatically incorporates continuity of tractions on $y=0$, as well as displacements outside the slip zones. Thus there only remains to consider the normal tractions

$$
N(x)=\sigma_{y y}(x, 0)+\tau_{y y}(x, 0)
$$

and the shearing tractions

$$
S(x)=\sigma_{x y}(x, 0)+\tau_{x y}(x, 0)
$$

in the slip zones. They must satisfy at all times the conditions

$$
\begin{gathered}
|S(x)|=f|N(x)| \\
N(x)<0 \\
\operatorname{sgn} S(x)=\operatorname{sgn} \frac{\mathrm{d} h(x)}{\mathrm{d} t}
\end{gathered}
$$

in these zones.

Due to the symmetry of the problem, two slip zones symmetrically placed, as shown in Fig. 1, will appear when (4) is violated. The location and extent of the slip zones are determined by the parameters $b$ and $c$ which are unknowns. Predicting the algebraic sign of $S(x)$ from the Flamant solution, (16) can be replaced with

$$
S(x)=f \operatorname{sgn} x N(x), \quad b<|x|<c
$$

and (18) with

$$
\operatorname{sgn} S(x)=\operatorname{sgn} h(x), \quad b<|x|<c
$$

for monotonically increasing $P(t)$. However, (19) must be verified a posteriori.

The symmetry of the problem implies that

$$
B_{x}(x)=B_{x}(-x)
$$

and consequently (19) yields the integral equation

$$
\int_{b}^{c} \frac{B_{x}(\xi)}{\xi-x} \mathrm{~d} \xi+\int_{b}^{c} B_{x}(\xi) k_{1}(x, \xi) \mathrm{d} \xi=\frac{\pi(\kappa+1)}{2 \mu}\left[f p_{0}-\frac{2 P a^{2}(x+f a)}{\pi\left(x^{2}+a^{2}\right)^{2}}\right], \quad b<x<c
$$

where

$$
\begin{aligned}
k_{1}(x, \xi)= & -\frac{1}{x+\xi}+\frac{x-\xi}{4 a^{2}+(x-\xi)^{2}}+\frac{x+\xi}{4 a^{2}+(x+\xi)^{2}}-\frac{12 a^{2}(x-\xi+2 f a)}{\left[4 a^{2}+(x-\xi)^{2}\right]^{2}} \\
& -\frac{12 a^{2}(x+\xi+2 f a)}{\left[4 a^{2}+(x+\xi)^{2}\right]^{2}}+\frac{64 a^{4}(x-\xi+2 f a)}{\left[4 a^{2}+(x-\xi)^{2}\right]^{3}}+\frac{64 a^{4}(x+\xi+2 f a)}{\left[4 a^{2}+(x+\xi)^{2}\right]^{3}}
\end{aligned}
$$


In addition we must require that

$$
\int_{b}^{c} B_{x}(\xi) \mathrm{d} \xi=0
$$

in order to have slip restricted to the two zones $b<|x|<c$.

The Cauchy singular integral equation (22), subject to condition (24), must be solved numerically for $B_{x}(x)$. Once this is done, the shearing tractions in both the slip and stick zones follow as

$$
S(x)=-\frac{2 P}{\pi a} \frac{a^{3} x}{\left(a^{2}+x^{2}\right)^{2}}+\frac{2 \mu}{\pi(\kappa+1)}\left\{\int_{b}^{c} \frac{B_{x}(\xi)}{x-\xi} \mathrm{d} \xi+\left.\int_{b}^{c} B_{x}(\xi) k_{1}(x, \xi)\right|_{f=0} \mathrm{~d} \xi\right\}
$$

The normal tractions are similarly

$$
N(x)=-p_{0}+\frac{2 P}{\pi a} \frac{a^{4}}{\left(a^{2}+x^{2}\right)^{2}}+\frac{2 \mu}{\pi(\kappa+1)} \int_{b}^{c} B_{x}(\xi) k_{2}(x, \xi) \mathrm{d} \xi
$$

where

$$
k_{2}(x, \xi)=-\frac{24 a^{3}}{\left[4 a^{2}+(x-\xi)^{2}\right]^{2}}-\frac{24 a^{3}}{\left[4 a^{2}+(x+\xi)^{2}\right]^{2}}+\frac{128 a^{5}}{\left[4 a^{2}+(x-\xi)^{2}\right]^{3}}+\frac{128 a^{5}}{\left[4 a^{2}+(x+\xi)^{2}\right]^{3}} .
$$

\section{NUMERICAL SOLUTION AND RESULTS}

Putting the integral equation (22) in a dimensionless form by the change of variables

where

$$
\xi / a=\delta r+\sigma, \quad x / a=\delta s+\sigma
$$

we obtain

$$
\delta=(c-b) / 2 a, \quad \sigma=(c+b) / 2 a
$$

$\frac{1}{\pi} \int_{-1}^{1} \frac{B_{x}(r)}{r-s} \mathrm{~d} r+\frac{1}{\pi} \int_{-1}^{1} B_{x}(r) K(r, s) \mathrm{d} r=\frac{P(\kappa+1)}{2 \mu a}\left\{\frac{f p_{0} a}{P}-\frac{2}{\pi} \frac{\delta s+\sigma+f}{\left[(\delta s+\sigma)^{2}+1\right]^{2}}\right\}, \quad-1<s<1$.

The regular kernel $K(r, s)$ follows readily from (23), (28) and (29), and we refrain from recording the result. Equation (24) becomes

$$
\int_{-1}^{1} B_{x}(r) \mathrm{d} r=0
$$

For the numerical integration of (30) we follow the method of Erdogan and Gupta[17]. Since $B_{x}$ is a bounded function, we set[18]

where

$$
B_{x}(r)=\frac{P(\kappa+1)}{2 \mu a} w(r) \varphi(r)
$$

$$
w(r)=\left(1-r^{2}\right)^{1 / 2}
$$

The discretized form of $(30)$ becomes

$$
\sum_{i=1}^{n} \frac{1-r_{i}^{2}}{n+1} \varphi\left(r_{i}\right)\left[\frac{1}{r_{i}-s_{k}}+K\left(r_{i}, s_{k}\right)\right]=\frac{f}{\lambda}-\frac{2}{\pi\left[\left(\delta s_{k}+\sigma\right)^{2}+1\right]^{2}}, \quad k=1, \ldots, n+1
$$

where

$$
\begin{array}{ll}
r_{i}=\cos \left(\frac{i \pi}{n+1}\right), & i=1, \ldots, n \\
s_{k}=\cos \left[\frac{i(2 k-1)}{2(n+1)}\right], & k=1, \ldots, n+1
\end{array}
$$


and

$$
\lambda=P / p_{0} a
$$

is a dimensionless loading parameter. Note that the consistency condition that must be satisfied for a bounded solution[19] is already included in (34). Finally, the discretized form of (31) becomes

$$
F=\sum_{i=1}^{n}\left(1-r_{i}^{2}\right) \varphi\left(r_{i}\right)=0
$$

The unknown parameters $\delta$ and $\sigma$ make the system (34) and (38) nonlinear. To simplify the iteration procedure we specify $\delta$, while leaving $\sigma$ and the loading parameter $\lambda$ as unknowns. With $\delta$ fixed, we guess a value for $\sigma$ and choose $n$ equations from the system (34) to solve for $\varphi\left(r_{i}\right)$. The remaining equation in (34) is used to determine $\lambda$. We then substitute into (38), which in general is not satisfied. We next iterate for $\sigma$ until $F$ is made to vanish. In practice, we choose $n$ as an even number and use the $(1 / 2 n+1)$ equation in (34) to solve for $\lambda$. Once the $n$ values of $\varphi\left(r_{i}\right)$ are obtained, we compute the discrete values of $B_{x}(r)$ from (32), and $h(x)$ from (13) using the trapezoidal rule of integration. The shearing tractions are obtained from the discretized form of (25). In the slip zones, (25) contains a Cauchy integral and the method of Erdogan and Gupta must be used, but in the stick zones the choice of the collocation points is open. The normal tractions in the slip zones are obtained from the shearing tractions on account of the boundary condition (19), and in the stick zones from the discretized form of (26).

Finally we must also check the various unilateral conditions. It was verified numerically in all cases considered that the normal tractions remained compressive, that

$$
|S(x)|<f|N(x)|
$$

in the stick zones, and that also (20) is satisfied. The reason why (20) implies (18) for a monotonically increasing concentrated force becomes clear once the solution is constructed: Both $S(x)$ and $h(x)$ are multiplied by $P(t)$ and, if (20) holds, (18) is satisfied as long as $P(t)$ and $\mathrm{d} P(t) / \mathrm{d} t$ are of the same sign.

The extent and location of the slip zones for various values of the friction coefficient are shown in Fig. 2 as functions of the loading parameter $\lambda$ for a tensile concentrated force. It may be noted that the curves intersect the vertical axis at the separation load. The tangential shift, and the normal and shearing tractions are depicted in Figs. 3-5 for $\delta=0.2$. The corresponding results for a compressive force are presented in Figs. 6-9. The shearing tractions do not show much variation with friction and are only plotted for $f=0.25$. It may also be remarked that the normal tractions are hardly affected by slip and are practically the same as in the bilateral problem.

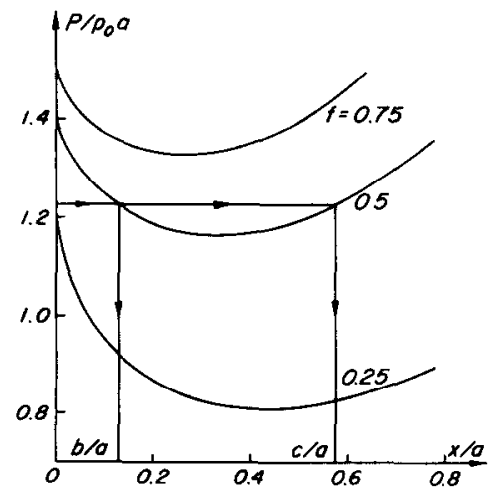

Fig. 2.

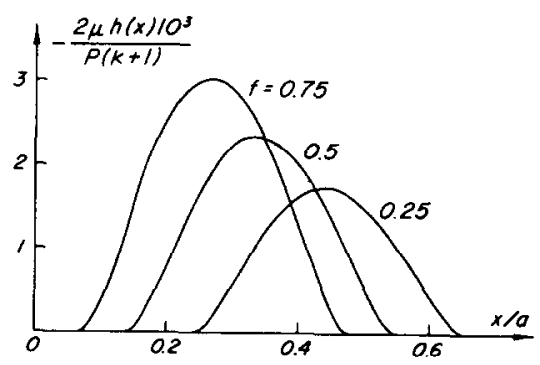

Fig. 3.

Fig. 2. Extent and location of the slip zones for a tensile force and different coefficients of friction.

Fig. 3. Tangential shift for tensile force and $\delta=0.2$. 

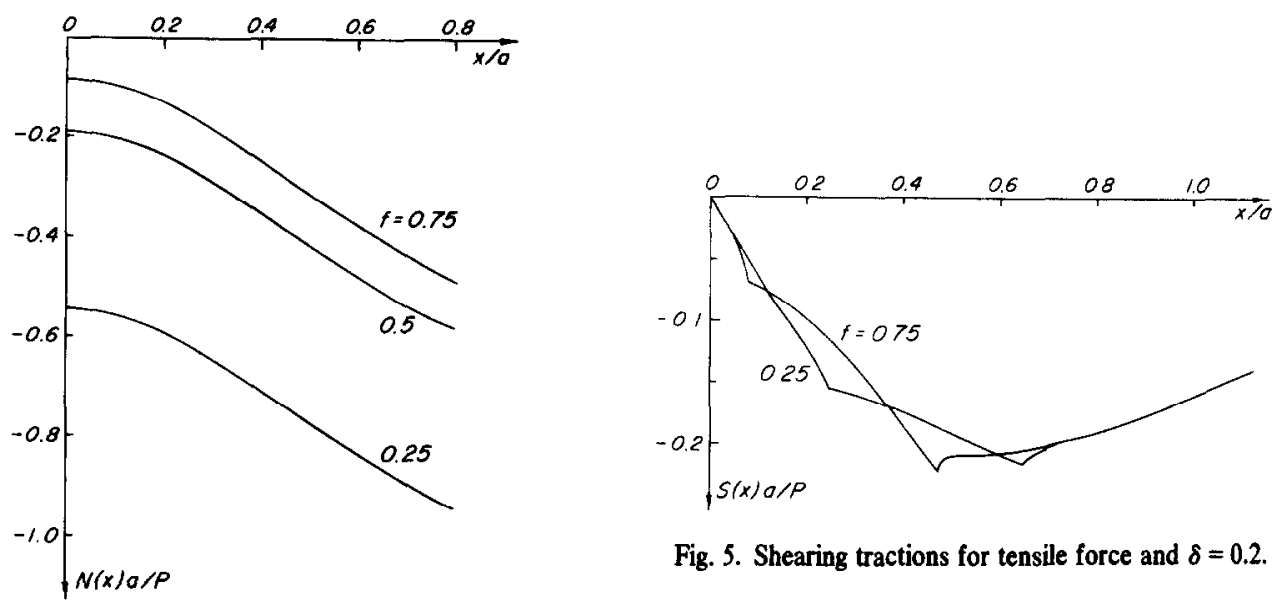

Fig. 5. Shearing tractions for tensile force and $\delta=0.2$.

Fig. 4. Normal tractions for tensile force and $\delta=0.2$.
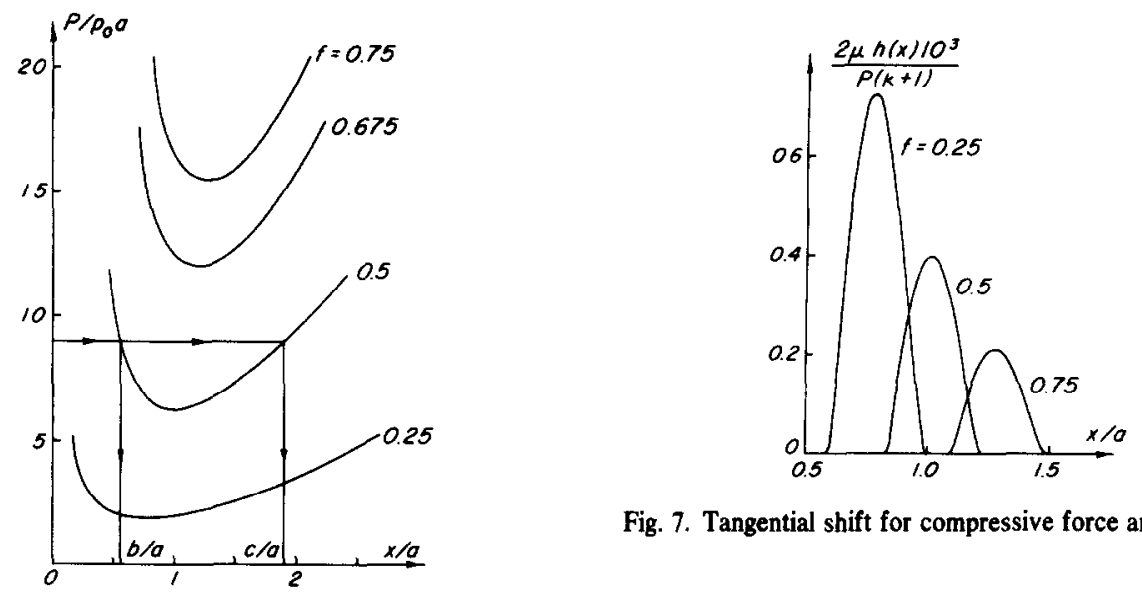

Fig. 7. Tangential shift for compressive force and $\delta=0.2$.

Fig. 6. Extent and location of the slip zones for a compressive force and different coefficients of friction.
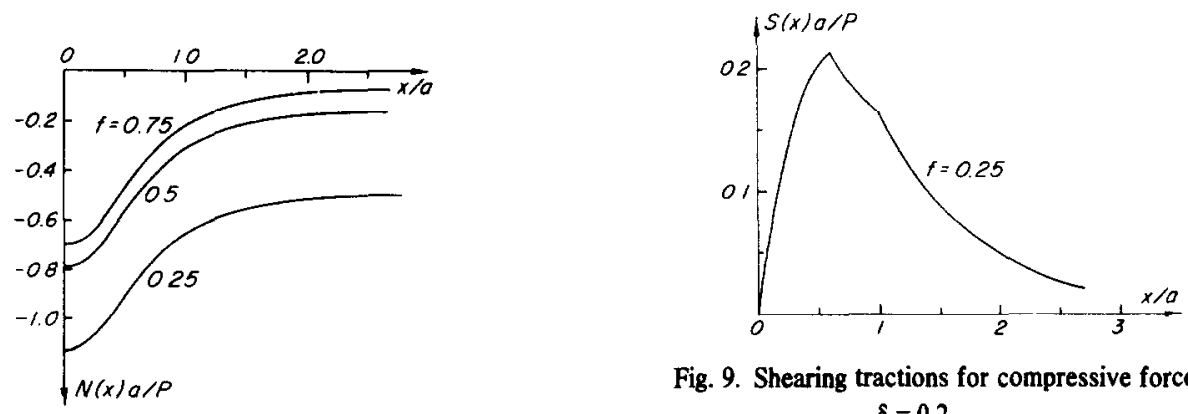

Fig. 9. Shearing tractions for compressive force and $\delta=0.2$.

Fig. 8. Normal tractions for compressive force and $\delta=0.2$.

The case of the layer and substrate consisting of different materials can be treated in a similar fashion. The only difference is that the solutions of the bilateral and dislocation problems would have to be constructed by means of Fourier transforms. The counterparts of $(1,2)$ and $(10,11)$ then are infinite integrals which will enter the right side and the regular kernel of the governing integral equation (22).

Acknowledgement-One of the authors (M. C.) wishes to acknowledge the support by the National Science Foundation under the grant ENG 77-25302.

\section{REFERENCES}

[1] J. DUNDURS, In The Mechanics of the Contact Between Deformable Bodies (Edited by A. D. de Pater and J. J. Kalker), pp. 54-66. Delft University Press (1975).

[2] L. M. KEER, J. DUNDURS and K. C. TSAI, J. Appl. Mech. 39, 1115 (1972). 
[3] L. M. KEER and K. CHANTARAMUNGKORN, J. Elasticity 2, 191 (1972).

[4] M. RATWANI and F. ERDOGAN, Int. J. Solids Structures 9, 921 (1973).

[5] K. C. TSAI, J. DUNDURS and L. M. KEER, J. Appl. Mech. 41, 703 (1974).

[6] M. B. CIVELEK and F. ERDOGAN, Int. J. Solids Structures 10, 639 (1974).

[7] F. ERDOGAN and M. RATWANI, J. Appl. Mech. 41, 673 (1974).

[8] J. DUNDURS, K. C. TSAI and L. M. KEER, J. Appl. Mech. 42, 221 (1975).

[9] M. B. CIVELEK and F. ERDOGAN, J. Appl. Mech. 42, 136 (1975).

[10] M. B. CIVELEK and F. ERDOGAN, J. Appl. Mech. 43, 175 (1976).

[11] M. R. GECIT and F. ERDOGAN, Int. J. Solids Structures 14, 771 (1978).

[12] M. B. CIVELEK, F. ERDOGAN and A. O. CAKIROGLU, Int. J. Engng Sci. 16, 669 (1978).

[13] S. P. TIMOSHENKO and J. N. GOODIER, Theory of Elasticity, 3rd Edn. McGraw-Hill, New York (1970).

[14] J. DUNDURS and T. MURA, J. Mech. Phys. Solids 12, 177 (1964).

[15] J. DUNDURS and G. P. SENDECKYJ. J. Appl. Phys. 36. 3353 (1965).

[16] J. DUNDURS, In Mathematical Theory of Dislocations (Edited by T. Mura, pp. 70-115). American Society of Mechanical Engineers (1969).

[17] F. ERDOGAN and G. GUPTA, Quart. Appl. Math. 29, 525 (1972).

[18] J. DUNDURS and M. COMNINOU, J. Elasticity 9, 71 (1979).

[19] N. I. MUSKHELISHVILI, Singular Integral Equations. Noordhoff, Leyden (1953).

(Received 10 May 1979) 